\title{
Scientists, keep an open line of communication with the public
}

\author{
The COVID-19 pandemic has opened up a direct channel between scientists and the public. Keeping it open must \\ become part of scientists' mission.
}

T he COVID-19 pandemic has thrust many researchers and the work they do into the public spotlight. From Twitter threads to the opinion pages of major news outlets, scientists are out of the lab and are engaging directly with the general public. Public awareness and interest in medical research is soaring, fueled in part by prompt access to the scientific literature through preprint servers and free access to content that in the past sat behind paywalls, and in part by the ever-growing use of social-media platforms as vehicles for highlighting, sharing and discussing new results. As traditional communication barriers are broken down, not only among scientists, but especially between them and the general public, researchers must embrace the opportunity to reach beyond the walls of their labs and rethink the role they want to play in society at large.

Increased engagement with the public can be powerful, but it also poses many challenges and comes with enormous responsibility. Researchers seldom receive training on how to communicate outside of the academic environment, nor are they incentivized to interact and learn from professional science communicators. Studies suggest that the academic attitude toward science communication has not substantially changed for the past 30 years. Moreover, traditional approaches to communicating with the public lack meaningful engagement and need to move beyond the paternalistic views of scientific communication, wherein researchers talk at the public rather than engaging them in an active dialogue. This model is counterproductive to effective messaging and can discourage public involvement and trust in research endeavors. This has been seen in past surveys showing that only $10 \%$ of the British public would describe themselves as "confident engagers" in science - including clinical trials. Instead, researchers should be pursing an ongoing dialogue that empowers the public to be actively involved in science, as key stakeholders. Such an approach recognizes and honors the public in a respectful manner and creates more effective connections that can be built upon. However, this is also challenging, as it forces researchers to gain a deeper understanding of public responses to complicated, emotionally charged scientific issues. For example, members of the public may already have an appropriate understanding of the scientific facts and concepts on an issue, yet struggle or disagree with the implications. Therefore, researchers need to be better listeners, an issue raised in a recent UK poll of the public's attitude toward science, so that questions and concerns can be better understood and addressed.

Improving education is key to overcoming these challenges. The academic community needs to recognize that the 21st-century scientist must be able to effectively communicate with many different audiences - from specialists to the lay person. Graduate and postdoctoral programs must ensure that their students and trainees have access to the tools and training needed to develop those skills. Importantly, this training needs to have the goal of training researchers to be eloquent listeners, sympathetic and adaptive to the needs of the public rather than superior and inflexible. Furthermore, young researchers starting their careers need institutional support and guidance from professional science communicators to fine-tune their skills and to pass those on to their trainees. Equipping researchers to engage with the general public should not be seen as a substitute for the role of professional scientific communicators but a complement to it.

The rewards of public engagement in research are worth the effort. A recent meta-analysis of over 25 different studies showed that increased patient and public involvement in clinical trials significantly improves participant rates of enrollment and retention. In the study, researchers highlight the importance of public engagement in these trials, characterizing it as being carried out 'with' or 'by' patients and/or members of the public rather than 'to', 'about' or 'for' them. It recognizes that the effectiveness of these trials is strongest when people with lived experience of the condition being studied are involved as research partners. This supports the view of patients and the public as experience-based experts who can contribute knowledge that is complementary to that of scientists. The impact of engaging patients as partners in research is explored in a special feature on Patient-led Research in this issue of Nature Medicine.

Social media is also proving to be a powerful tool with which researchers can communicate directly with the public in an accessible way, allowing them to inform the community about clinical trials and vaccine research, for instance. Perhaps more importantly, researchers have found social media effective in recruiting broader and more-diverse populations for clinical trials, including historically hard-to-reach populations, such as young cancer survivors, Latino men who have sex with men and the Deaf community. Through increased engagement, researchers can expand the reach of their work and increase its societal value.

While the COVID-19 pandemic has catalyzed direct engagement between scientists and the public, it is critical that this communication channel remains open going forward. Researchers must build upon this experience to establish a continuous, long-lasting dialogue with the general public. The purpose for scientific research is not only to gain new knowledge but also to put that knowledge to use to benefit all sectors of society. Engagement with the public not only strengthens public trust in science but ultimately serves to raise awareness and education of science in such a way that is mutually beneficial to all stakeholders. Science is far too important of a societal good to remain confined within the walls of the lab.

Published online: 7 October 2020 https://doi.org/10.1038/s41591-020-1111-1 